\title{
Irreversibility and rate dependence in sheared adhesive suspensions
}

\author{
Zhouyang Ge $\odot,{ }^{1,2, *}$ Raffaella Martone, ${ }^{3}$ Luca Brandt $\odot,{ }^{1}$ and Mario Minale $\odot^{3, \dagger}$ \\ ${ }^{1}$ Department of Engineering Mechanics, KTH Royal Institute of Technology, SE-100 44 Stockholm, Sweden \\ ${ }^{2}$ Department of Mechanical Engineering, University of British Columbia, Vancouver, British Columbia, \\ Canada V6T $1 Z 4$ \\ ${ }^{3}$ Department of Engineering, University of Campania “Luigi Vanvitelli”, Real Casa dell'Annunziata, via \\ Roma 29-81031 Aversa, Italy
}

(Received 8 June 2020; revised 27 June 2021; accepted 27 September 2021; published 13 October 2021)

\begin{abstract}
Recent experiments report that slowly sheared noncolloidal particle suspensions unexpectedly exhibit rate $(\omega)$-dependent complex viscosities in oscillatory shear, despite a constant relative viscosity in steady shear. Using a minimal hydrodynamic model, we show that van der Waals attraction gives rise to this behavior. At volume fractions $\phi=20-50 \%$, the complex viscosities in both experiments and simulations display power-law reductions in shear, with a $\phi$-dependent exponent maximum at $\phi=40 \%$, resulting from the interplay between hydrodynamic, collision, and adhesive interactions. Furthermore, this rate dependence is accompanied by diverging particle diffusivities and pronounced cluster formations after repeated oscillations. Previous studies established that suspensions transition from reversible absorbing states to irreversible diffusing states when the oscillation amplitude exceeds a $\phi$-dependent critical value $\gamma_{0, \phi}^{c}$. Here, we show that a second transition to irreversibility occurs below an $\omega$-dependent critical amplitude, $\gamma_{0, \omega}^{c} \leqslant \gamma_{0, \phi}^{c}$, in the presence of weak attractions.
\end{abstract}

DOI: 10.1103/PhysRevFluids.6.L101301

The flow properties of suspensions remain challenging to predict despite the tremendous progress to date. Even the simplest suspensions, consisting of non-Brownian particles suspended in a densitymatching Newtonian fluid, while exhibiting a Newtonian behavior in steady shear (SS) flow, can show very rich phenomena under oscillatory shear (OS), such as flow irreversibility and chaos [1], absorbing state transitions [2,3], and microstructure reorganizations at large accumulated strains [4,5]. All these fundamental behaviors are predicted using very few ingredients in the equations of motion that are in order: hydrodynamic forces, including lubrication between adjacent particles, and hard-sphere collisions. Because the latter do not possess any characteristic time scale and their amplitude is proportional to the driving hydrodynamic force, the system and all its material functions are rate independent $[6,7]$.

So far, it has been assumed that the suspension microstructure depends on the strain amplitude $\left(\gamma_{0}\right)$ and strain history $\left(\gamma_{\text {tot }}\right)$ in OS, and rate dependence (if any) is manifested in both SS and OS. However, recent experiments challenge this assumption and demonstrate a frequency $(\omega)$-dependent

\footnotetext{
*zhoge@mech.kth.se

†mario.minale@unicampania.it
}

Published by the American Physical Society under the terms of the Creative Commons Attribution 4.0 International license. Further distribution of this work must maintain attribution to the author(s) and the published article's title, journal citation, and DOI. Funded by Bibsam. 

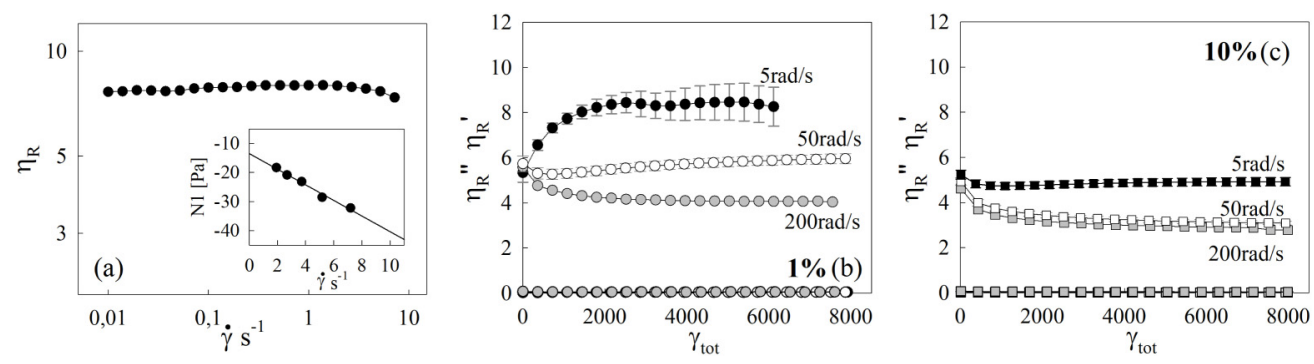

FIG. 1. Experiment at $\phi=40 \%$ : (a) Relative viscosity $\left(\eta_{R}\right)$ and first normal stress difference $\left(N_{1}\right.$, inset) vs shear rate in SS. (b),(c) Evolution of the dynamic viscosity $\left(\eta_{R}^{\prime}\right)$ and its elastic counterpart $\left(\eta_{R}^{\prime \prime}\right)$ vs the total accumulated strain at $\gamma_{0}=1 \%$ (b) and $10 \%$ (c) in OS. The angular frequencies are indicated in the figure.

rheology in OS in the absence of any rate dependence in SS [8-10]. These authors demonstrate that, in OS, the suspension viscosity only depends on the maximum shear rate $\left(\gamma_{0} \omega\right)$ and that data taken at different volume fractions $(\phi)$ can be rescaled on a single master curve, so as to highlight a universal behavior of these materials. Furthermore, this rheological observation questions the physics of self-organization in the simplest driven noncolloidal suspension: it was assumed that suspensions undergo transitions from reversible absorbing states to irreversible chaotic states if $\gamma_{0}$ exceeds a $\phi$-dependent critical amplitude $\gamma_{0}^{c}(\phi)$ independent of the driving frequency $[1,2,11]$; now the experiments in [10] imply that $\omega$ may also affect irreversibility.

In this Letter, we combine experiments with simulations and show that a weak van der Waals attraction is enough to induce the sought rate dependence in OS, while keeping the SS behavior rate independent. Moreover, we reveal that this rheological behavior in OS is accompanied by enhanced particle diffusivities and cluster formations below a critical shear rate, thus the onset of rate dependence when reducing $\gamma_{0}$ is closely related to the threshold for irreversibility. A deeper understanding of self-organization and dynamical phase transition in suspensions is not only of fundamental interest [12-15], but has also attracted practical attention due to its applications in hyperuniform photonic materials or suspension flow control [16-18]. This Letter shows that a physical ingredient up to now neglected, i.e., interparticle attraction, must be taken into account.

Our experimental samples consist of glass hollow microspheres $(5-50 \mu \mathrm{m}$ in diameter, mean $15.4 \mu \mathrm{m}$ ) dispersed in a low molecular weight polyisobutene, which is known to behave as a Newtonian fluid (viscosity $15.8 \mathrm{Pas}$ at $21.2^{\circ} \mathrm{C}$ ) at the shear rates of interest, at three volume fractions $(20-40 \%)$. Time sweep oscillatory tests are executed on a constant-strain rheometer, ARES G2 (TA Instruments), equipped with a cone-and-plate geometry, by imposing a sinusoidal strain during each run, $\gamma(t)=\gamma_{0} \sin (\omega t)$, for $\gamma_{0}$ from $0.5 \%$ to 2 and $\omega$ from 5 to $200 \mathrm{rad} / \mathrm{s}$. After a steady preconditioning shear, the complex viscosity is followed in time in units of the total accumulated strain, $\gamma_{\text {tot }}=4 \gamma_{0} n_{\text {cyc }}$, where $n_{\text {cyc }}$ is the number of cycles of the oscillatory shear. All tests are performed repeatedly according to standard protocols, cf. Ref. [10]. Further details on the experimental procedure can also be found in Ref. [19].

The suspensions are inertialess and non-Brownian, as the particle Reynolds number is smaller than $10^{-6}$ and the Péclet number is larger than $10^{5}$. Particle sedimentation can be neglected, as the average Shields number is about $10^{3}$. A characteristic time arising from Brownian diffusion or sedimentation is thus irrelevant in the investigated suspensions and, accordingly, the SS behavior shows constant viscosity and first normal stress difference negative and linear in the shear rate; see Fig. 1(a). In OS, the relative complex viscosity, $\eta_{R}^{*} \equiv \eta_{R}^{\prime}-i \eta_{R}^{\prime \prime}$, evolves in time and is a function of $\gamma_{0}$, as widely reported in the literature $[2,4,20]$, but, surprisingly, it also decreases with $\omega$ (i.e., shear thinning). To illustrate this, we display in Figs. 1(b) and 1(c) the relative dynamic $\left(\eta_{R}^{\prime}\right)$ and elastic $\left(\eta_{R}^{\prime \prime}\right)$ viscosities vs $\gamma_{\text {tot }}$, for two values of $\gamma_{0}$ and three of $\omega$ at $\phi=40 \%$ (data for other $\phi$ are available in Ref. [10]). $\eta_{R}^{\prime}$ is always about two orders of magnitude larger than $\eta_{R}^{\prime \prime}$, thus it is practically coincident with $\eta_{R}^{*}$, highlighting the viscous behavior of the suspension. In general, an 
$\omega$-dependent regime is observed for $\gamma_{0} \lesssim 1$ and an $\omega$-independent regime otherwise. These two regimes coincide with those observed by Lin et al. [21], who showed that in the first regime the microstructure self-arrangement is driven by shear-induced particle diffusions, while in the second one the microstructure is immediately formed by the oscillation itself, similarly to what happens for a steady flow reversal that is indeed rate independent. The dependence on the frequency and the importance of diffusion indicate that a nonhydrodynamic force is at play [22], which cannot be hard-sphere interactions. To identify this force, we now turn to numerical simulations.

Discrete element simulations based on a minimal hydrodynamic model are performed for suspensions at zero Reynolds number [23]. Specifically, our model determines particle nonaffine trajectories according to lubrication, contact (hard-sphere), and interparticle potentials, similarly to the Stokesian dynamics [24]. Since the full many-body hydrodynamic interactions are truncated at the level of lubrication, our method is mostly accurate for dense suspensions where interparticle gaps are small, cf. Refs. [25,26]. The results below are therefore for $\phi=40 \%$ and 50\% [27].

To begin with, we note that several forces have been linked in the literature to rate dependence (shear thinning in particular) in dense, non-Brownian suspensions: (normal-)load-dependent friction [28], electrostatic repulsion [25,29], and van der Waals attraction [30,31]. Friction, as well as any other possible dissipation mechanisms due to surface roughness, should lead to no less rheological response in SS than OS, since larger deformations activate more frictional contacts at the same $\phi$. Indeed, we have checked that including the contact model of [28] in our system gives shear thinning in SS instead of OS, contrary to what the experiments show. Therefore, an explanation in terms of friction is unlikely.

As for electrostatic repulsion, its impact on the suspension rheology can be understood as an effective volume effect [29]. At low shear (thus stress), few particle pairs come closer than an enlarged radius due to the finite-range repulsive force; the portion of such particles (thus the effective $\phi)$ reduces with the shear, leading to shear thinning. Although plausible, it is difficult to rigorously establish this argument in all scenarios. In fact, our simulations show that electrostatic repulsion causes shear thickening in OS, again contradicting the experimental results [32].

The only possibility left is attraction. Here, we consider the simplest van der Waals (vdW) attraction, which is always present regardless of material or particle size, although weak for micron-size glass $[33,34]$. Let us underline that the glass beads used in the experiments are typically considered as insert particles and that the use of small vdW forces does not break this paradigm, as they are very often negligible with respect to the other forces acting on the particles. As in Ref. [31], we model a nonretarded and additive vdW as $F_{\mathrm{vdw}}=A \bar{a} / 12\left(h^{2}+\epsilon^{2}\right)$, where $A$ is the Hamaker constant, $\bar{a}=2 a_{i} a_{j} /\left(a_{i}+a_{j}\right)$ is the harmonic mean radius of two interacting particles, $h$ is their surface gap, and $\epsilon$ is a small constant to prevent divergence of $F_{\mathrm{vdw}}$ at $h=0$; we use $\epsilon=\sqrt{10^{-5}} a$ to model glass beads. The maximal attraction $\mathcal{F}=A a / 12 \epsilon^{2}$ introduces a characteristic stress scale, $\tau_{\mathcal{F}}=\mathcal{F} / \pi a^{2}$, for two touching particles, opposed to the minimal shear stress, $\tau_{s}=\eta_{0} \gamma_{0} \omega / 2 \pi\left(\eta_{0} \dot{\gamma}\right.$ in SS), where $\eta_{0}$ denotes the solvent viscosity. The ratio $\tau_{s} / \tau_{\mathcal{F}}$ thus defines a nondimensional shear rate, hereafter denoted $\mathrm{Sr} \equiv 6 \eta_{0} \epsilon^{2} a \gamma_{0} \omega / A$.

Figure 2 shows the rheology of this model adhesive system. Remarkably, with the addition of only a weak vdW attraction $(\propto 1 / \mathrm{Sr})$, the essential features of the complex rate-(in)dependent behaviors in (SS) OS seen in experiments are captured numerically [35]. Specifically, below a critical shear rate in OS (the dotted line in Fig. 2), $\eta_{R}^{*}$ exhibits power-law reductions with shear, $\eta_{R}^{*} \sim \mathrm{Sr}^{-\alpha}$, with $\alpha$ a $\phi$-dependent positive exponent largest at $\phi=40 \%$. This nonmonotonic dependence can be understood by considering the limits of very dilute and highly packed suspensions: at vanishing volume fractions, collisions and vdW attractions are negligible, and the system is dominated by hydrodynamic forces and therefore nearly rate independent; close to packing, lubrication and contact forces dominate but, because both are proportional to the shear rate, the system again exhibits weak rate dependence.

To provide quantitative evidence, we display the different contributions to the relative viscosity for the ten representative cases in Fig. 3. The budget terms correspond to the Stokes (stk), lubrication 


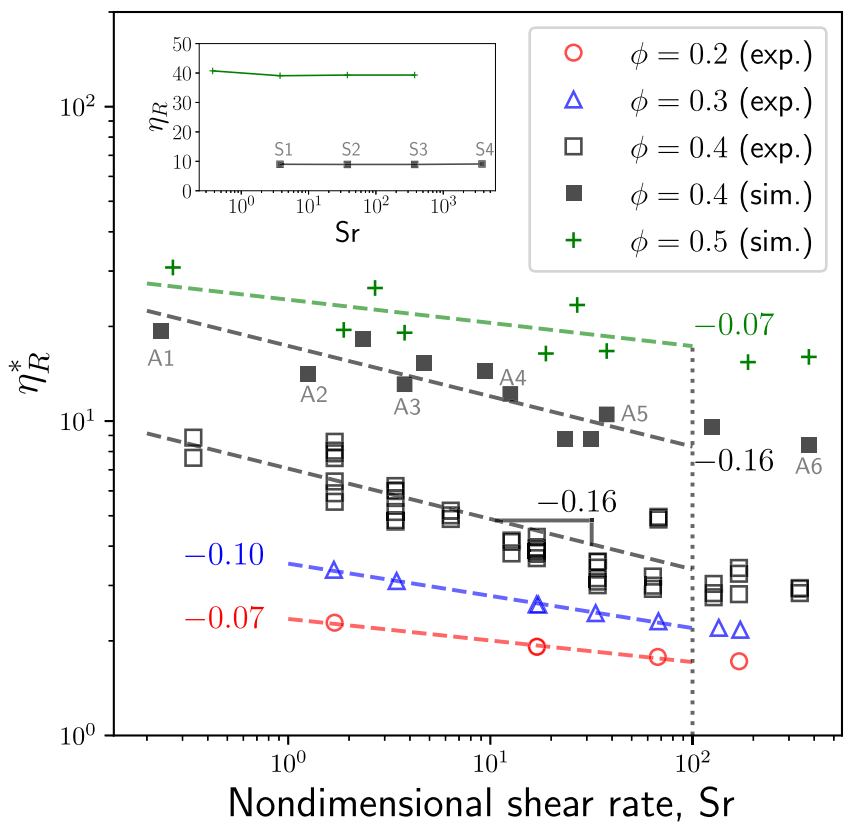

FIG. 2. Suspension viscosities in OS and SS (inset). Dashed lines are least squares fits to data for $\mathrm{Sr}<100$; numbers indicate the slopes.

(lub), contact (ctt), and vdW forces extracted from the simulations [23]. Surprisingly, at $\phi=40 \%$, where the maximum vdW effect is expected, virtually no contributions from the attractive vdW forces are visible across three decades of Sr. In SS, the lubrication and contact stresses are constant (stk only depends on $\phi$ thus identical in all cases) and so the relative viscosity; in OS, however, lubrication is shear dependent while contact stresses are vanishing. Since the rate dependence results from vdW, naively, one would expect it also makes a rate-dependent contribution to the stress budget, or at least be active in OS. Its absence implies that the rheology is indirectly modulated by weak attractions. The question, then, is how such indirect modulation occurs.

Recall that, without any inertial, thermal, or non-Newtonian effects, the rheological properties of a suspension at any given time are determined solely from its underlying microstructure [34]. The microstructure evolution is mediated by time-reversible Stokes flows, though the particle dynamics themselves satisfy Onsager's variational principle for general irreversible processes [36]. Indeed, both experiments and simulations have shown that particle diffusion can occur in slowly sheared suspensions, leading to irreversible and ultimately chaotic dynamics $[1,20,22,37,38]$. Particularly,

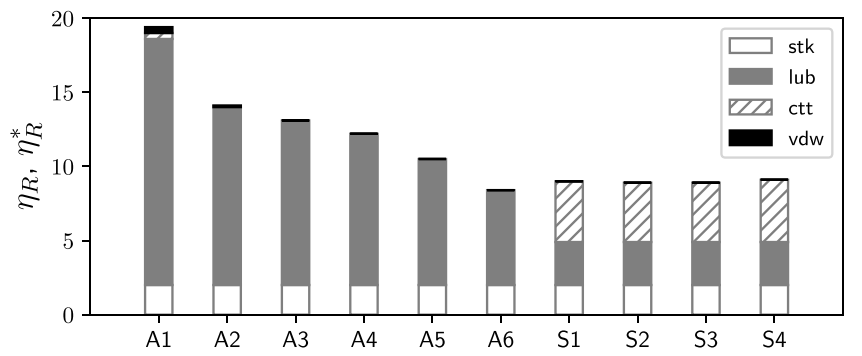

FIG. 3. Shear stress components of representative OS and SS cases at $\phi=40 \%$; see Fig. 2 for the annotation. 

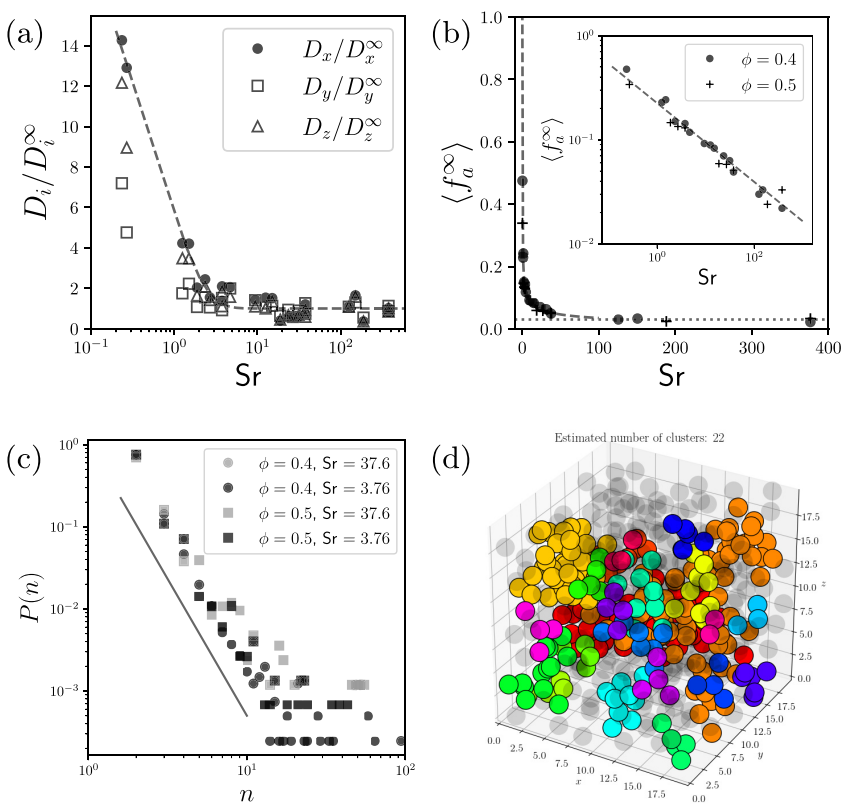

(d)

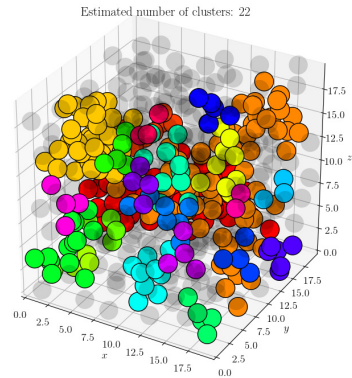

FIG. 4. Microstructure statistics in OS. (a) Relative particle diffusivities at $\phi=40 \%$ and $50 \%$ (not distinguished). The dashed line is a fit of $D_{x} / D_{x}^{\infty}$. (b) Mean fraction of active (colliding) particles on linear and logarithmic (inset) scales. The dashed line is a power-law fit with slope $\beta=-0.40$. (c) Power-law distribution of cluster size, $P(n) \sim n^{-3.33}$ (solid line). (d) A snapshot of the particle suspension at $\phi=40 \%$. Different cluster groups are indicated by colors.

in OS, the threshold for irreversibility was found to be gauged by a $\phi$-dependent critical strain amplitude $\gamma_{0}^{c}(\phi)$ : for $\gamma_{0}>\gamma_{0}^{c}$, suspensions are irreversible with nonvanishing effective diffusivities, $D_{i}=\left\langle\left(\Delta_{i} / a\right)^{2}\right\rangle / 2 \gamma_{\text {tot }}\left(\Delta_{i}\right.$ denotes the particle displacement in any spatial direction $i$ at integerperiod intervals); for $\gamma_{0}<\gamma_{0}^{c}$, the dynamics evolve towards absorbing states with $D_{i} \approx 0$ in finite time. The system undergoes a continuous phase transition (likely conserved directed percolation [15]) at $\gamma_{0}=\gamma_{0}^{c}$, indicated by a nonzero order parameter $\left\langle f_{a}^{\infty}\right\rangle$, defined as the mean fraction of "actively" colliding particles. This theoretical framework [2] applies to a remarkable variety of situations; see, e.g., Ref. [15] and references therein. We note that none of them invokes a role for frequency, though.

It is therefore natural to examine the microstructure statistics of our suspensions. Figure 4 shows the numerical results at $\phi=40 \%$ and 50\%. Surprisingly, at strain amplitudes well under the irreversibility threshold just mentioned $\left(\gamma_{0} \leqslant 0.2\right.$ for all simulations with $\gamma_{0}^{c} \approx 0.82$ at $\phi=40 \%$ [39]), our suspensions can be both irreversible and active. This irreversibility only occurs below a fixed critical shear rate $\mathrm{Sr}_{c}$. Figure 4(a) shows that, by renormalizing the effective diffusivities with their averages in the infinite shear limit, e.g., $D_{x}^{\infty}=\left\langle D_{x}\right\rangle_{\mathrm{Sr} \rightarrow \infty}$, all values at $\phi=40 \%$ and $50 \%$ diverge with reducing Sr. A similar behavior is observed for the order parameter, which diverges below $\operatorname{Sr}_{c}$ as $\left\langle f_{a}^{\infty}\right\rangle \sim \mathrm{Sr}^{\beta}$, with $\beta=-0.40$ [Fig. 4(b)]. Akin to the self-organized criticality due to slow sedimentation [3], particles in adhesive suspensions actively form clusters with a near power-law size distribution [Fig. 4(c)]. As an example, Fig. 4(d) shows a snapshot of the suspension at $\phi=40 \%$, where various particle clusters are displayed in color (inactive particles are displayed in grey).

Comparison of Figs. 4(a), 4(b) and 2 indicates that this new threshold for irreversibility is closely related to the onset of rate dependence, both at $\mathrm{Sr}_{c} \approx 100$ [40]. Below $\mathrm{Sr}_{c}$, the microstructure is indirectly modulated by weak vdW interactions; their relative intensity is inversely proportional to 

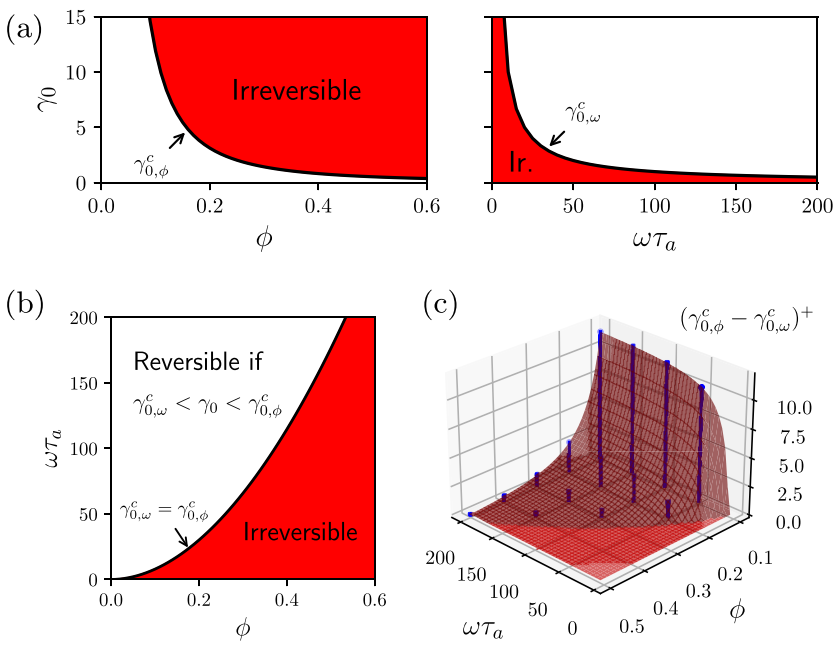

FIG. 5. Phase diagrams for irreversibility in sheared adhesive suspensions. (a) Critical strain amplitudes due to collisions (left) or attractions (right). $\omega$ is normalized by $\tau_{a} \equiv 6 \eta_{0} \epsilon^{2} a / A$. (b) Projection of the irreversibility map on the $\left(\phi, \omega \tau_{a}\right)$ plane. (c) Volume of the reversible absorbing states in the $\left(\phi, \omega \tau_{a}, \gamma_{0}\right)$ space.

the shear rate, thus the suspension shows diffusive dynamics and rate dependence at low $\gamma_{0}$. Above $\mathrm{Sr}_{c}$, hydrodynamic interactions overcome $\mathrm{vdW}$, resulting in time-reversible Stokesian dynamics and a rate-independent suspension rheology. Here, we cannot determine the value of $\mathrm{Sr}_{c}$ exactly due to the increasing uncertainties in the data near it. Nevertheless, by matching experiments and simulations at $\mathrm{Sr}_{c}=100$ (Fig. 2), we estimate the Hamaker constant of the vdW interaction to be $A \approx 4 \times 10^{-19} \mathrm{~J}$ in the system under investigation. This is within the range of $A$ for most condensed phases (cf. Ref. [33], p. 254), which validates our assumption.

More importantly, the existence of a unique $\mathrm{Sr}_{c}$ suggests that there is a frequency-dependent critical amplitude for the transitions between absorbing and diffusing states. This can be written as $\gamma_{0, \omega}^{c}=\operatorname{Sr}_{c} A / 6 \eta_{0} \epsilon^{2} a \omega$, which is independent of the volume fraction and thus must coexist with $\gamma_{0}^{c}(\phi)$ (hereafter $\gamma_{0, \phi}^{c}$ ). In between the two thresholds, i.e., $\gamma_{0, \omega}^{c}<\gamma_{0}<\gamma_{0, \phi}^{c}$, suspensions can reach reversible absorbing states from any initial condition; below and above this range, irreversibility arises from either attractions (low $\gamma_{0}$ ) or collisions (high $\gamma_{0}$ ); see Figs. 5(a) and 5(b). However, $\gamma_{0, \omega}^{c}$ may also exceed $\gamma_{0, \phi}^{c}$, resulting in irreversible dynamics for all $\gamma_{0}$. For example, consider a $40 \%$ suspension oscillating at $5 \mathrm{rad} / \mathrm{s}$ : if $\gamma_{0}>0.82$, the suspension is chaotic due to collisions [1,2]; whereas if $\gamma_{0} \leqslant 0.82$, it is irreversible due to attractions $\left(\gamma_{0, \omega}^{c}=18.8\right.$ for our suspensions). Note that, when $0.82<\gamma_{0}<18.8$, both these mechanisms promote irreversibility. In such a case, the dynamics are controlled by collisions because collision-induced diffusions at larger $\gamma_{0}$ occur over a shorter time scale. We have checked that the suspension relaxation time (i.e., the time to reach steady states) is always orders-of-magnitude longer in our simulations and experiments (cf. Fig. 1) than those in Ref. [2]. Thus, for large values of $\gamma_{0}$ the system dynamics are collision dominated: this finally explains why rate dependence is observed only for smaller $\gamma_{0}$ in OS, but not in SS, under the same shear rate.

A new phase diagram thus emerges for irreversibility in sheared adhesive suspensions. Figures 5(b) and 5(c) show the region of reversible steady dynamics in $\phi$ and $\omega$, and the size of the reversibility window in $\gamma_{0}$; the latter is measured by a positive difference between the two critical amplitudes, $\left(\gamma_{0, \phi}^{c}-\gamma_{0, \omega}^{c}\right)^{+}$, where $(x)^{+}=\max (x, 0)$. Notice how rapidly the window reduces as $\phi$ or $1 / \omega$ increases. Considering the chaotic nature of many-body systems (e.g., ideal gas, planets, etc.), microscopic irreversibility is the hallmark rather than an exception for suspensions. This is true even in Stokes flow for, whenever particles get close, their dynamics are not governed by the 
hydrodynamics alone. Here, by combining experiments with simulations, we have shown that there is a fundamental connection between irreversibility and suspension rheology, both of which depend on the strain amplitude as well as the driving frequency. Future work may examine this picture when including long-range hydrodynamic interactions, or explore the so-called active fluids whose intriguing rheologies defy reversibility even without passive particles [41].

We thank R. Radhakrishnan, C. Ness, F. Peters, A. Los, J. Chun, and A. Leshansky for helpful discussions. The work was supported by the Swedish Research Council (Grant No. VR 2014-5001) and University of Campania "L. Vanvitelli" under the program "VALERE: VAnviteLli pEr la RicErca" project, SEND.

[1] D. J. Pine, J. P. Gollub, J. F. Brady, and A. M. Leshansky, Chaos and threshold for irreversibility in sheared suspensions, Nature (London) 438, 997 (2005).

[2] L. Corté, P. M. Chaikin, J. P. Gollub, and D. J. Pine, Random organization in periodically driven systems, Nat. Phys. 4, 420 (2008).

[3] L. Corté, S. J. Gerbode, W. Man, and D. J. Pine, Self-Organized Criticality In Sheared Suspensions, Phys. Rev. Lett. 103, 248301 (2009).

[4] J. M. Bricker and J. E. Butler, Oscillatory shear of suspensions of noncolloidal particles, J. Rheol. 50, 711 (2006).

[5] J. M. Bricker and J. E. Butler, Correlation between stresses and microstructure in concentrated suspensions of non-brownian spheres subject to unsteady shear flows, J. Rheol. 51, 735 (2007).

[6] E. J. Hinch, The measurement of suspension rheology, J. Fluid Mech. 686, 1 (2011).

[7] R. N. Chacko, R. Mari, S. M. Fielding, and M. E. Cates, Shear reversal in dense suspensions: the challenge to fabric evolution models from simulation data, J. Fluid Mech. 847, 700 (2018).

[8] C. Carotenuto, M. C. Merola, and M. Minale, Effect of frequency on the complex viscosity of a concentrated non-brownian suspension, in Times of Polymers (TOP) and Composites 2014: Proceedings of the 7th International Conference on Times of Polymers (TOP) and Composites, AIP Conf. Proc. No. 1599, edited by A. D'Amore, D. Acierno, and L. Grassia (AIP, Melville, NY, 2014), p. 258.

[9] R. Martone, L. P. Paduano, C. Carotenuto, and M. Minale, Dependence of suspension complex viscosity on frequency: Strain-controlled vs. stress-controlled tests, in 9th International Conference on "Times of Polymers and Composites": From Aerospace to Nanotechnology, AIP Conf. Proc. No. 1981, edited by A. D’Amore, D. Acierno, and L. Grassia (AIP, Melville, NY, 2018), p. 020185.

[10] R. Martone, C. Carotenuto, and M. Minale, Non-brownian newtonian suspensions may be rate dependent in time sweep oscillatory shear flow, J. Rheol. 64, 1075 (2020).

[11] P. Pham, J. E. Butler, and B. Metzger, Origin of critical strain amplitude in periodically sheared suspensions, Phys. Rev. Fluids 1, 022201(R) (2016).

[12] G. I. Menon and S. Ramaswamy, Universality class of the reversible-irreversible transition in sheared suspensions, Phys. Rev. E 79, 061108 (2009).

[13] G. Düring, D. Bartolo, and J. Kurchan, Irreversibility and self-organization in hydrodynamic echo experiments, Phys. Rev. E 79, 030101(R) (2009).

[14] J. R. Royer and P. M. Chaikin, Precisely cyclic sand: Self-organization of periodically sheared frictional grains, Proc. Natl. Acad. Sci. USA 112, 49 (2015).

[15] C. Ness and M. E. Cates, Absorbing-State Transitions In Granular Materials Close To Jamming, Phys. Rev. Lett. 124, 088004 (2020).

[16] D. Hexner and D. Levine, Hyperuniformity Of Critical Absorbing States, Phys. Rev. Lett. 114, 110602 (2015).

[17] S. Wilken, R. E. Guerra, D. J. Pine, and P. M. Chaikin, Hyperuniform Structures Formed By Shearing Colloidal Suspensions, Phys. Rev. Lett. 125, 148001 (2020). 
[18] C. Ness, R. Mari, and M. E. Cates, Shaken and stirred Random organization reduces viscosity and dissipation in granular suspensions, Sci. Adv. 4, eaar3296 (2018).

[19] C. Carotenuto, G. Rexha, R. Martone, and M. Minale, The microstructural change causing the failure of the cox-merz rule in newtonian suspensions: experiments and simulations, Rheol. Acta 60, 309 (2021).

[20] V. Breedveld, D. van den Ende, R. Jongschaap, and J. Mellema, Shear-induced diffusion and rheology of noncolloidal suspensions: Time scales and particle displacements, J. Chem. Phys. 114, 5923 (2001).

[21] Y. Lin, N. Phan-Thien, and B. C. Khoo, Short-term and long-term irreversibility in particle suspensions undergoing small and large amplitude oscillatory stress, J. Rheol. 57, 1325 (2013).

[22] G. Drazer, J. Koplik, B. Khusid, and A. Acrivos, Deterministic and stochastic behaviour of non-brownian spheres in sheared suspensions, J. Fluid Mech. 460, 307 (2002).

[23] Z. Ge and L. Brandt, Implementation note on a minimal hybrid lubrication/granular dynamics model for dense suspensions, arXiv:2005.12755.

[24] J. F. Brady and G. Bossis, Stokesian dynamics, Annu. Rev. Fluid Mech. 20, 111 (1988).

[25] R. Mari, R. Seto, J. F. Morris, and M. M. Denn, Shear thickening, frictionless and frictional rheologies in non-brownian suspensions, J. Rheol. 58, 1693 (2014).

[26] O. Cheal and C. Ness, Rheology of dense granular suspensions under extensional flow, J. Rheol. 62, 501 (2018).

[27] All simulations consider bidisperse suspensions of 500 spheres, with size ratio 1.4, in a cubic box subject to Lees-Edwards boundary condition. In OS, only small amplitudes are used, $\gamma_{0}=0.05-0.2$, while frequency is controlled by adjusting the nondimensional time scale.

[28] L. Lobry, E. Lemaire, F. Blanc, S. Gallier, and F. Peters, Shear thinning in non-brownian suspensions explained by variable friction between particles, J. Fluid Mech. 860, 682 (2019).

[29] G. Chatté, J. Comtet, A. Niguès, L. Bocquet, A. Siria, G. Ducouret, F. Lequeux, N. Lenoir, G. Ovarlez, and A. Colin, Shear thinning in non-brownian suspensions, Soft Matter 14, 879 (2018).

[30] E. Brown, N. A. Forman, C. S. Orellana, H. Zhang, B. W. Maynor, D. E. Betts, J. M. DeSimone, and H. M. Jaeger, Generality of shear thickening in dense suspensions, Nat. Mater. 9, 220 (2010).

[31] A. Singh, S. Pednekar, J. Chun, M. M. Denn, and J. F. Morris, From Yielding To Shear Jamming In A Cohesive Frictional Suspension, Phys. Rev. Lett. 122, 098004 (2019).

[32] Z. Ge, R. Martone, L. Brandt, and M. Minale, Weakly adhesive suspension shows rate-dependence in oscillatory but not steady shear flows, arXiv:2006.04494.

[33] J. N. Israelachvili, Intermolecular and Surface Forces (Academic, New York, 2011).

[34] J. Mewis and N. J. Wagner, Colloidal Suspension Rheology (Cambridge University Press, Cambridge, UK, 2012).

[35] The vertical discrepancy between simulations and experiments may be due to polydispersity of the glass beads or residual short-range repulsion forces that are not modeled numerically.

[36] M. Doi, Onsager's variational principle in soft matter, J. Phys.: Condens. Matter 23, 284118 (2011).

[37] E. C. Eckstein, D. G. Bailey, and A. H. Shapiro, Self diffusion of particles in shear flow of a suspension, J. Fluid Mech. 79, 191 (1977).

[38] D. Leighton and A. Acrivos, Measurement of shear-induced self-diffusion in concentrated suspensions of spheres, J. Fluid Mech. 177, 109 (1987).

[39] $\gamma_{0}^{c}(\phi)$ can be calculated from the power-law fit $C \phi^{-\alpha}$ reported in Ref. [1]. However, there is a typo for the value of $\alpha$. It should be 1.93 instead of -1.93 .

[40] In general, the onset of rate dependence may depend on $\phi$ through a critical suspension shear stress, $\sigma^{*} \sim \eta_{R}^{*}(\phi) \mathrm{Sr}_{c}$, since rheological quantities average over the entire suspension. In our case, $\eta_{R}^{*}(\phi)$ differs at most by a factor of 4 at large $\mathrm{Sr}$, thus we refrain from introducing a $\phi$ dependence in $\mathrm{Sr}_{c}$ for simplicity.

[41] D. Saintillan, Rheology of active fluids, Annu. Rev. Fluid Mech. 50, 563 (2018). 